\title{
Estigma hacia el aborto y sus consecuencias: acciones para reducirlo
}

\author{
Fabiola Orihuela-Cortés y Ma. Luisa Marván
}

\begin{abstract}
Resumen
El estigma es la devaluación social que vive una persona por poseer características que van en contra de las normas culturales establecidas. El aborto, a pesar de ser un procedimiento muy común, está sumamente estigmatizado debido a que, en el entendimiento colectivo, se tiene como una práctica moralmente equivocada y socialmente inaceptable. En el presente artículo, se abordarán los diferentes niveles de la sociedad en los que se manifiesta este estigma, como lo son: el nivel de los medios de comunicación y opinión pública, el nivel estructural o legal, el nivel institucional, el nivel comunitario, y el nivel individual. De igual manera, es importante conocer las diversas acciones que realiza la sociedad civil para reducir el estigma hacia el aborto, por lo que se enlistan distintas asociaciones que tienen como propósito la reivindicación del aborto mediante grupos de reflexión, cursos, campañas, videos, talleres, entre otras acciones. Por último, se proponen algunas acciones individuales que se pueden realizar con el fin de reducir el estigma entorno al aborto.
\end{abstract}

Palabras clave: estigma, aborto inducido, consecuencias del estigma, reducción del estigma.

\section{STIGMA TOWARDS ABORTION AND ITS CONSEQUENCES: ACTIONS TO REDUCE IT}

\begin{abstract}
Stigma is the social devaluation that a person experiences by possessing characteristics contrary to those culturally established. Despite abortion being a common procedure, it is extremely stigmatized. The stigma towards abortion is based on the collective understanding that termination of pregnancy is a morally wrong and socially unacceptable practice. In this article will be addressed the different facets of society in which this stigma manifests itself such as: in media and public opinion, at structural or legal level, at institutional level, at community level, and at individual level. It is important to know the actions that civil society is taking to reduce the stigma towards abortion, for this reason, various associations that have as purpose the demand of abortion through reflection groups, courses, campaigns, videos, workshops, among other actions are listed. Finally, some individual actions are proposed that can be carried out to reduce the stigma towards abortion.
\end{abstract}

Keywords: stigma, induced abortion, consequences of stigma, reduction of stigma.

Recepción: 12/02/2021. Aprobación: 15/03/2021. http://doi.org/10.22201/cuaieed.16076079e.2021.22.4.7 
"Estigma hacia el aborto y sus consecuencias: acciones para reducirlo"

Fabiola Orihuela-Cortés y Ma. Luisa Marván

Vol. 22, Núm. 4, julio-agosto 2021

Revista Digital Universitaria

Mtra. Fabiola Orihuela-Cortés

fabiola.oc7@gmail.com

orcid.org/0000-0002-2614-6273

Feminista y activista por los derechos sexuales y reproductivos de las mujeres. Cuenta con una licenciatura y una maestría en Psicología, ambas realizadas en la Universidad Veracruzana. Actualmente, estudia un doctorado en Psicología en la misma institución. Ha colaborado en investigaciones sobre actitudes hacia el aborto y la sexualidad adolescente. Además, ha facilitado grupos de reeducación para mujeres que han vivido violencia de pareja, y se ha desempeñado como docente de licenciatura en Psicología.

Dra. Ma. Luisa Marván

mlmarvan@gmail.com orcid.org/0000-0002-0670-2260

Psicóloga con doctorado en Ciencias Biomédicas otorgado por la Universidad Nacional Autónoma de México, unam. Se ha dedicado cerca de 30 años a la actividad académica, tanto en la docencia como en la investigación. Actualmente, es investigadora del Instituto de Investigaciones Psicológicas de la Universidad Veracruzana y pertenece al Sistema Nacional de Investigadores. Ha publicado numerosos artículos a nivel nacional e internacional sobre diversos temas de salud reproductiva, como el aborto. 
El aborto voluntario o inducido es la interrupción del embarazo antes de que el embrión o feto sea viable fuera del útero. A nivel mundial, se estima que el 61\% de las mujeres que han tenido un embarazo no planeado deciden realizarse un aborto (Bearak et al., 2020). Se trata de un procedimiento tan frecuente que es probable que conozcas a una mujer que haya abortado, e incluso, si eres mujer, seas una de ellas.

Sin embargo, es poco frecuente que las personas hablen libremente sobre experiencias relacionadas con el aborto. Esto se debe, en gran medida, a que las mujeres que han decidido interrumpir su embarazo suelen ser estigmatizadas. El estigma es una construcción social que se basa en los estereotipos culturales que existen en la sociedad, y que recae sobre las personas con características que van en contra de las normas establecidas en una cultura. Estas características no son ni buenas ni malas, solamente son diferentes a lo establecido (Zamberlin, 2015). El estigma hacia la mujer que se ha realizado un aborto está basado en el entendimiento colectivo de que el aborto es una práctica moralmente equivocada e inaceptable (Cockrill y Herold, 2013). Esto se manifiesta desde comentarios y comportamientos de rechazo hacia las mujeres que han interrumpido voluntariamente su embarazo, hasta regulaciones restrictivas en torno al aborto (Zamberlin, 2015).

\section{¿Cómo puedo identificar el estigma hacia el aborto?}

El estigma hacia el aborto se manifiesta en diversas esferas de la sociedad, y de acuerdo con Kumar et al., (2009) se presenta en los siguientes niveles (imagen 1):

\section{Esferas donde se manifiesta el estigma hacia el aborto}

Imagen 1. Esferas donde se manifiesta el estigma hacia el aborto. Adaptación del modelo de Kumar et al., (2009). Elaboración propia.

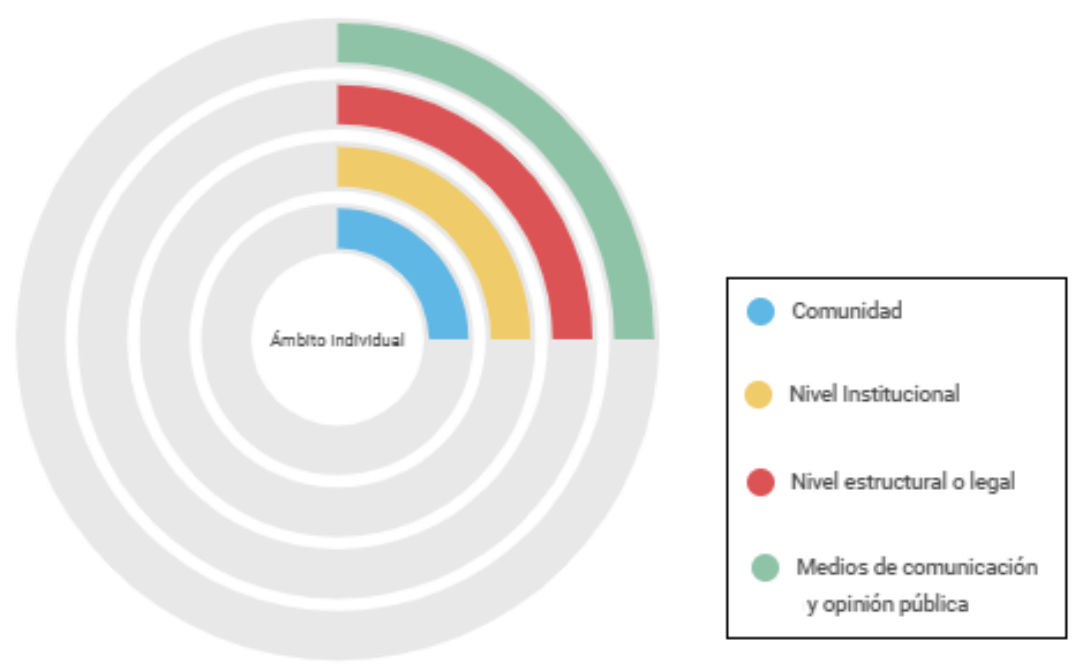




\section{Medios de comunicación y opinión pública}

En nuestro país, encontramos discursos negativos hacia el aborto que suelen señalar a las mujeres que han interrumpido voluntariamente su embarazo como personas promiscuas, irresponsables, indeseables, poco confiables y pecadoras (Sorhaindo et al., 2014; Sorhaindo et al., 2016). Hasta, en ocasiones, también son tachadas de criminales (Lamas, 2014). Además, existe un discurso que le adjudica vida al embrión para señalar y culpar a las mujeres que deciden abortar (Bosio, 2018). Inclusive, se creó el término síndrome post aborto, para nombrar a los problemas psicológicos severos que presentaban las mujeres ha causa de la interrupción voluntaria de su embarazo. Sin embargo, este síndrome ha sido desmentido por la Asociación Americana de Psicología (APA) en dos ocasiones (Maroto, 2009).

Asimismo, es frecuente encontrar personas afuera de las clínicas en donde se realizan abortos en la Ciudad de México para amedrentar a las mujeres por su decisión de interrumpir voluntariamente su embarazo (Lamas, 2014). Además, se han creado centros de asesoría en diferentes ciudades del país con el fin de persuadir a las mujeres que desean abortar (Morán-Faundes, 2015). También, en el 2020 se realizaron eventos masivos a favor de la "protección de la vida desde la concepción hasta la muerte natural" como el Congreso Iberoamericano por la Vida y la Familia en el que participaron grupos representantes de México; la ix Marcha por la Vida en México celebrada a través de redes sociales; y las Caravanas por la Vida convocadas en Quintana Roo y en Puebla.

\section{Nivel estructural o legal}

A pesar de que el aborto está muy restringido legalmente en México, existen diferentes condiciones en cada estado, llamadas causales, por las que se permite abortar de manera legal. Sólo hay una causal por la que el aborto es legal en todo el país, cuando el embarazo es producto de una violación. Además, únicamente en dos estados se permite libremente el aborto, siempre y cuando se lleve a cabo en las primeras 12 semanas de gestación: en la Ciudad de México se despenalizó en abril de 2007 y en Oaxaca en septiembre de 2019. Estos hechos evidencian la criminalización social del aborto inducido en nuestro país. De igual manera, representa un acto de discriminación jurídica, y un obstáculo para que las mujeres puedan acceder a una atención médica adecuada que les permita abortar de manera legal y segura (Grupo de Información en Reproducción Elegida [GIRE], 2018; Ipas, 2017).

Además, en este nivel, existen varios casos en los que las autoridades criminalizan y perseguen de manera ilegal a mujeres que han tenido abortos espontáneos, es decir, involuntarios. Inclusive, a algunas de estas mujeres se les ha condenado a varios años de prisión. También, se ha negado el aborto legal en casos en los que el embarazo es producto de una violación. Esto es alarmante porque, como ya se mencionó, el aborto es legal en todo el país por esta causal (GIRE, 2018). En otra vertiente, a pesar de que el aborto es un tema de salud 
pública, llama la atención que hay pocas campañas publicitarias oficiales para informar en qué consiste el aborto legal y seguro, así como de las opciones para acceder a éste (Ipas, 2017).

\section{Nivel institucional}

A pesar de la legalidad del aborto por la causal de violación en todo el país, y de su despenalización en dos estados, en los seguros médicos no está incluido el pago de la interrupción del embarazo cuando se lleva a cabo en hospitales privados (Lamas, 2014). Por otro lado, en el currículum de carreras como medicina y enfermería existe poco entrenamiento sobre el procedimiento para llevar a cabo un aborto inducido de forma segura (Zamberlin, 2015).

Otro aspecto importante es que, en ocasiones, el personal de salud muestra actitudes discriminatorias hacia las mujeres que acuden a los hospitales en búsqueda de una interrupción legal de su embarazo (Ipas, 2017). Más aún, es frecuente que el personal de salud apele a la objeción de conciencia, el derecho que tienen para no realizar un aborto si éste va en contra de sus creencias y valores personales (Rincón y Mazo, 2014). En este sentido, algunos autores han concluido que hay profesionales de la salud que apelan a la objeción de conciencia, no porque el aborto vaya en contra de sus creencias, sino para evitar ser estigmatizados (Lamas 2014; Rincón y Mazo, 2014).

\section{Nivel comunitario}

Las mujeres que han abortado son criticadas debido a las normas sociales que consideran a la maternidad como un aspecto sumamente importante en la vida de las mujeres. Debido a estas normas, se observa al aborto como algo negativo. Estas creencias suelen ser más comunes en personas de edad avanzada, con niveles altos de religiosidad, con pocos estudios académicos, y en personas que tienen un mayor número de hijos (LeTourneau, 2016; Sorhaindo et al., 2014; Sorhaindo et al., 2016).

\section{Ámbito individual}

El estigma en este nivel suele presentarse de tres maneras:

1) En un estigma internalizado: donde la mujer incorpora las creencias negativas que la sociedad ha establecido sobre el aborto, lo que la lleva a sentirse culpable y avergonzada por su decisión de interrumpir su embarazo (Cockrill y Nack, 2013).

2) En un estigma percibido: la mujer que ha abortado percibe ciertas actitudes negativas y comportamientos discriminatorios por parte de la sociedad.

3) En un estigma experimentado: la mujer tiene la experiencia real de ser objeto de agresiones, discriminación y hostigamiento por parte de otras personas (Cockrill y Nack, 2013). 


\section{¿Qué consecuencias tiene el estigma hacia el aborto?}

El estigma relacionado con el aborto se presenta hacia todas las personas involucradas en el procedimiento. Sin embargo, las mujeres que interrumpen su embarazo suelen ser quienes sufren, en un mayor grado, las consecuencias de este estigma. Debido a que en México sólo está despenalizado el aborto en dos estados, las mujeres con recursos económicos se pueden trasladar a una de esas ciudades para interrumpir su embarazo de manera legal y segura. Sin embargo, la mayoría de las mujeres no tienen las posibilidades para hacerlo y pueden verse presionadas para continuar con su embarazo sin desearlo, o para realizarse un aborto de manera clandestina, arriesgándose a tener un aborto inseguro (Ipas, 2017; Zamberlin, 2015).

Es importante mencionar que la mayoría de los abortos realizados bajo los protocolos recomendados por organismos como la Organización Mundial de la Salud (oms) son seguros, pero los abortos realizados por personal poco calificado, con métodos incorrectos, o en condiciones insalubres son un riesgo para la salud de la mujer (Sigh et al., 2018). Estas características sociales provocan la marginación social, médica y legal de la atención del aborto y son una barrera para que todas las mujeres puedan tener acceso a una interrupción del embarazo segura y de calidad. De igual manera, estos aspectos amenazan a su bienestar físico y mental (Cockrill, y Nack, 2013; O'Donell et al., 2018; Shellenberg et al., 2011), e incluso atentan contra la vida de las mujeres que deciden interrumpir su embarazo (Sigh et al. 2018).

Por otro lado, como ya se mencionó, muchas mujeres suelen sentirse culpables y avergonzadas al interrumpir su embarazo (Cockrill y Nack, 2013) y tratan de vivir en secreto su aborto, lo cual les genera tensión, angustia y aislamiento (Cedeño et al., 2019; Ipas, 2017; O’Donell et al. 2018). Además, el hecho de mantener un aborto en secreto afecta en otros dos aspectos: por un lado, orilla a las mujeres a elegir lugares que prometen un mayor grado de secretismo, en lugar de los que garantizan un aborto en condiciones óptimas; incluso hay mujeres que abortan sin ningún tipo de acompañamiento o de supervisión médica (Zamberlin, 2015). Por otro lado, el hecho de mantener en secreto un aborto, perpetua su estigmatización ya que parece que es un hecho que sólo ocurre esporádicamente (Shellenberg et al., 2011).

\section{¿Se podría reducir el estigma hacia el aborto?}

Es posible reducir los efectos negativos de la estigmatización en las mujeres después de haber abortado. Una manera de hacerlo es a través de círculos de reflexión en los que a las mujeres se les permite hablar libremente sobre su experiencia dentro de un ambiente positivo y de apoyo. En estos círculos también se busca generar confianza, crear conexiones y transformar su percepción negativa sobre el aborto a una positiva (Belfrage et al., 2020; Cockril y Biggs, 2018). 
"Estigma hacia el aborto y sus consecuencias: acciones para reducirlo"

Fabiola Orihuela-Cortés y Ma. Luisa Marván

Vol. 22, Núm. 4, julio-agosto 2021

Revista Digital Universitaria

Por otro lado, existen algunas organizaciones cuyo propósito es reducir el estigma hacia el aborto, entre ellas se encuentran las siguientes:

1) Católicas por el Derecho a Decidir (CDD): es una organización compuesta por personas católicas que defienden la laicidad del Estado, los derechos humanos, así como los derechos sexuales y reproductivos. CDD ha realizado campañas como: "Otra mirada católica del aborto", realizada en 2010 en donde se difundieron diversos argumentos sobre el aborto desde una mirada católica con el propósito de visualizar las diferentes posturas sobre el tema y que la Iglesia Católica no condena el aborto en ciertas circunstancias. Y "Catolicadas", que es una serie animada que trata diversos temas, y uno de ellos es la defensa del aborto seguro.

2) Ipas México: es una organización que realiza distintas actividades de sensibilización y capacitación en escuelas, colonias e instituciones, con el objetivo de visibilizar que las interrupciones del embarazo son más frecuentes de lo que creemos. Además, busca reducir el estigma relacionado con el aborto a través de diversos talleres.

3) Red Internacional por la Reducción de la Discriminación y el Estigma con relación al Aborto (INROADS): se trata de una comunidad virtual que brinda una serie de recursos y herramientas para ayudar a identificar el estigma hacia el aborto y para poder erradicarlo.

4) Acompañamiento Después de un Aborto Seguro (ADAS) y la Red por los Derechos Sexuales y Reproductivos (DDSER): son dos organizaciones mexicanas que realizan círculos de reflexión con el objetivo de reducir el estigma que viven las mujeres después de un aborto, específicamente, se busca disminuir su aislamiento.

5) El Fondo de Aborto para la Justicia Social María (Fondo María): busca, entre otras acciones, transformar el discurso negativo sobre el aborto a uno positivo a través de la campaña "Yo aborto, yo acompaño, yo transformo" en donde se da voz a los testimonios de las mujeres que han pasado por un aborto.

6) Instituto de Liderazgo Simone de Beauvoir (ILSB) y Redefine: lanzaron la campaña "Hablar es chido", la cual tiene la finalidad de difundir información sobre el aborto, generar charlas y foros de comunicación para que este tema se discuta abiertamente en la sociedad.

7) La Red por los Derechos Sexuales y Reproductivos en México (ANDAR) y La Sandía Digital: realizaron el cortometraje "Era yo, otra vez", con el cual se busca disminuir el estigma relacionado con el aborto al mostrar historias de tres mujeres de distintas generaciones en diversos contextos que han vivido un aborto inducido.

8) Focos: es una plataforma digital cuyo objetivo es visibilizar el aborto inducido como una práctica frecuente mediante testimonios confidenciales de mujeres (Imagen 2). 
Imagen 2. Marcha por el 28 de septiembre, Día de Acción Global por el Acceso al Aborto Legal y Seguro. Créditos: Surinam Arandia Azuara Ángeles

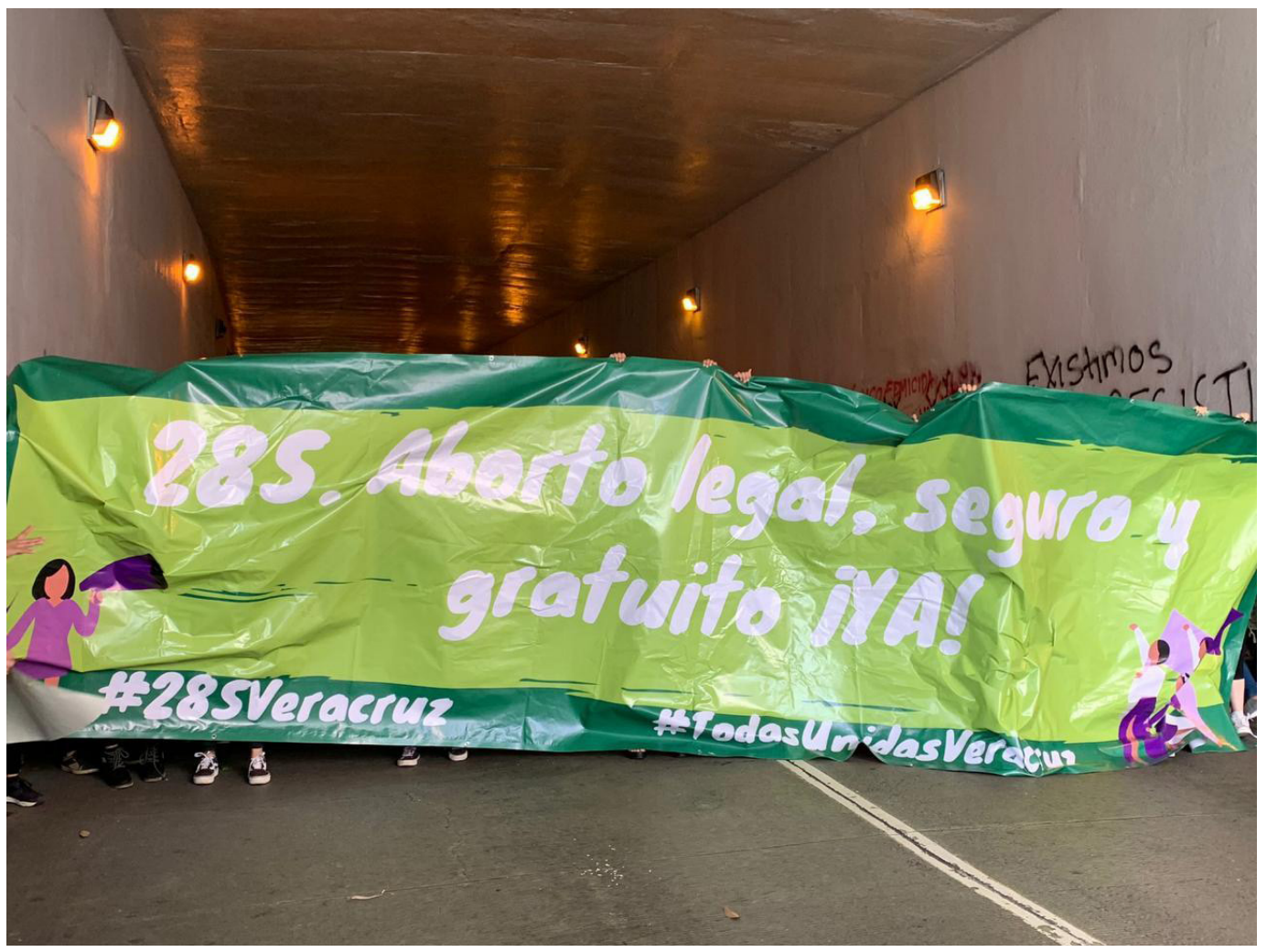

\section{¿Qué puedo hacer para reducir el estigma?}

Como se ha comentado, a pesar de ser un tema tabú, el aborto es una práctica frecuente. Por lo tanto, es importante que empecemos a verlo de manera natural y entendamos que las mujeres toman esta decisión por diversas razones y que todas son igual de válidas. Es necesario vernos como parte de una comunidad, por lo tanto, todos podemos agregar un granito de arena para reducir el estigma hacia el aborto y, con ello, las consecuencias adversas que éste trae a la vida de las mujeres.

Es necesario considerar que cuando una mujer se entera que está embarazada, puede o no reaccionar de manera positiva. Hay mujeres que no piensan que tener un hijo sea algo positivo en sus vidas, por lo que no podemos dar por hecho que todas las mujeres desean continuar con sus embarazos, y debemos respetar esta decisión. Además, es conveniente estar atentos a nuestro lenguaje, para que éste no sea estigmatizante, es decir, no utilizar expresiones que fomenten la estigmatización, por ejemplo: llamar a la mujer embarazada "madre", o al embrión/ feto "bebé" o "bebé no nacido". Más aún, hay que evitar felicitar a la mujer embarazada sin saber si está contenta con la noticia.

Además, si conocemos a una mujer que haya decidido interrumpir su embarazo, cuidemos no hacer comentarios negativos. Es importante tener en cuenta que sólo ella conoce su situación y sus circunstancias, y que cualquier 
decisión que haya tomado será la correcta, por eso debemos tener una postura de respeto. Si es preciso, podemos darle información sobre asociaciones civiles que pueden brindarle apoyo, por ejemplo: el Fondo María, la Red Necesito Abortar y aDAs Acompañamiento Emocional.

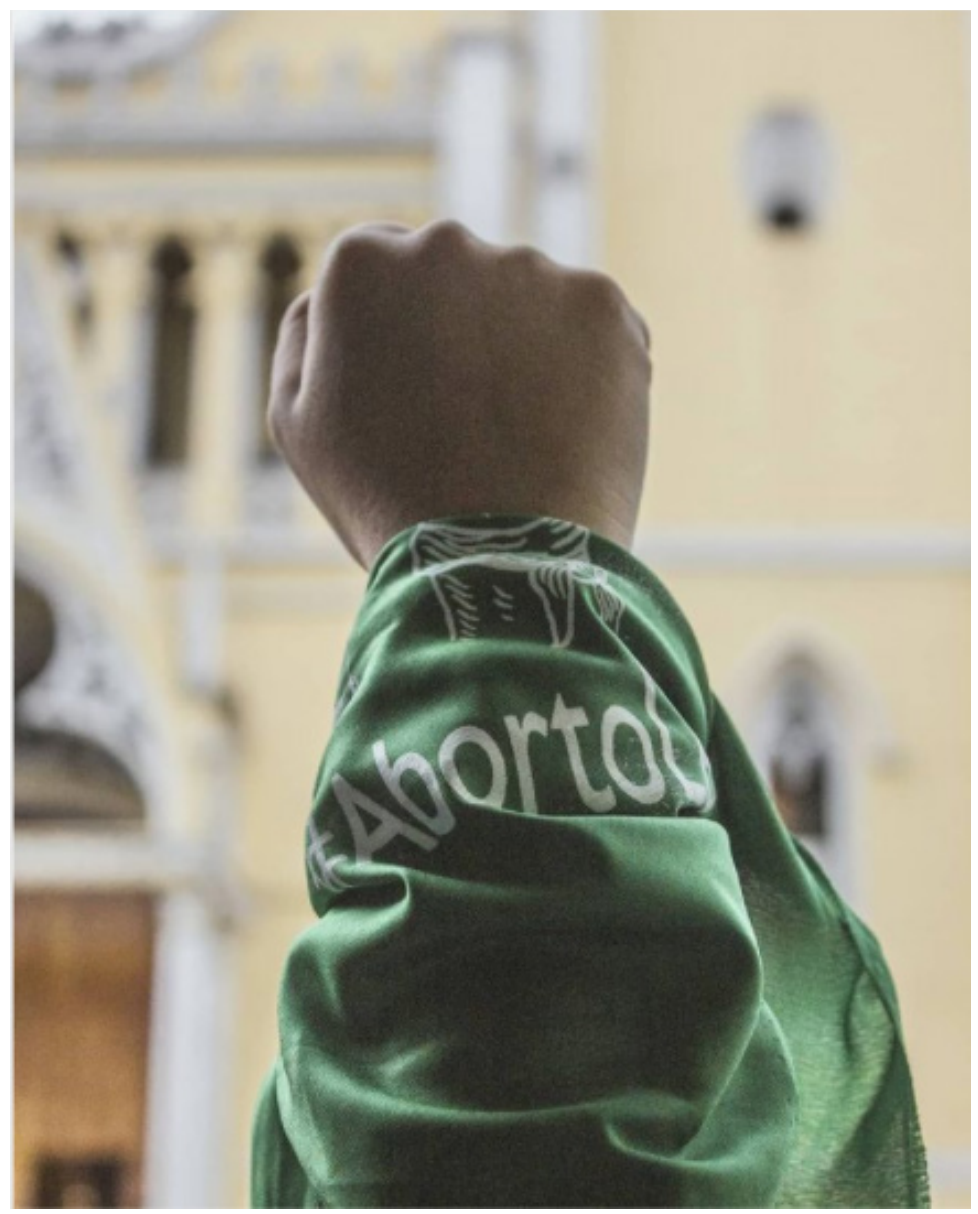

Por otro lado, la información que proviene de fuentes confiables siempre es una buena herramienta, así que podemos mantenernos informados sobre el aborto inducido para tener argumentos sólidos en contra de los mitos que existen alrededor de este procedimiento, por ejemplo, que abortar puede dejar infértil o propiciarle cáncer a la mujer. Además, es preciso familiarizarnos con los tratados internacionales y las normas oficiales que ratifican el aborto como un derecho de las mujeres, ya que, frecuentemente, no se aplican. Si todos conociéramos e hiciéramos válidas estas legislaciones, se ayudaría a reducir el estigma hacia el reconocimiento del aborto como un derecho de la mujer.

Otra acción que puede ayudar a disminuir este estigma es incluir los aspectos señalados en este artículo en pláticas con tus personas cercanas, incluso compartir publicaciones serias sobre el aborto en redes sociales. Esto nos ayuda a empezar a ver el aborto como un proceso normal en la vida de algunas mujeres y como una opción viable para todas. Ahora bien, si te interesa hacer más acciones, puedes acercarte a las distintas asociaciones que trabajan para orientar y ayudar a las mujeres que desean tener un aborto, o que

Imagen 3. Aborto Legal y Seguro. Créditos: Indra Cano trabajan con otros sectores de la sociedad para sensibilizar y concientizar sobre este tema con el fin de erradicar el estigma.

\section{Conclusión}

Es importante tener en cuenta que el contexto legal restrictivo del aborto no reduce su incidencia, ya que muchísimas mujeres han abortado a pesar de la penalización legal de este procedimiento. Mientras sigan existiendo embarazos no deseados, y, por consecuencia, abortos, es preciso que trabajemos en promover una despenalización "social" del aborto, es decir, en aceptar que el aborto inducido es un procedimiento frecuente y un derecho de las mujeres. Para lograr esto, es conveniente realizar distintas acciones a nivel individual y social que abonen a que las mujeres que interrumpen un embarazo puedan vivir este proceso sin estigma (Imagen 3). 


\section{Referencias}

- Bearak, J., Popinchalk, A., Ganatra, B., Moller, A., Tunçalp, Ö., Beavin, C., Kwok, L., y Alkema, L. (2020). Unintended pregnancy and abortion by income, region, and the legal status of abortion: estimates from a comprehensive model for 1990-2019. Lancet, 8.

* Belfrage, M., Ortiz-Ramirez, O., y Sorhaindo, A. (2019). Story Circles and abortion stigma in Mexico: a mixed-methods evaluation of a new intervention for reducing individual level abortion stigma. Culture, Health \& Sexuality. https://doi.org/10.108 0/13691058.2019.1577493

* Bosio, M. T. (2018). Eje Movimientos de mujeres y feministas-El derecho al aborto legal: una demanda que transversaliza los feminismos y desafía al Estado. Cuadernos de Coyuntura, (2), 89-93.

* Cedeño, L., Tena, O., Flores, F., y Rocha, T. (2019). Guardar/romper el silencio sobre la interrupción voluntaria del embarazo: narrativas de mujeres mexicanas. Athenea Digital, 19 (2).

* Cockrill, K., y Herold, S. (2013). Addressing abortion stigma through service delivery: a with paper. Ibis Reproductive Health. https://ibisreproductivehealth.org/ sites/default/files/files/publications/Addressing\%20abortion\%20stigma\%20 through\%20service\%20delivery.pdf

* Cockrill, K., y Nack, A. (2013). "I'm not that type of person": Managing the Stigma of Having an Abortion. Deviant Behavior, 34(12), 973-990.

* Cockrill, K. y Biggs, A. (2018). Can stories reduce abortion stigma? Findings from a longitudinal cohort study. Culture, health \& sexuality, 20(3), 335-350. https://doi.or g/10.1080/13691058.2017.1346202

* Grupo de Información en Reproducción Elegida (GIRE). (2018). Maternidad o castigo. La criminalización del aborto en México. https://gire.org.mx/blogs/maternidad-o-castigo/

- Ipas. (2017). ¿Qué es el estigma? El estigma relacionado con el aborto. https://www. ipasmexico.org/wp-content/uploads/2017/12/revistaEstigma-junio7.pdf

* Kumar, A., Hessini, L., y Mitchell, E. (2009). Conceptualising abortion stigma. Culture, Health y Sexuality,11(6),625- 639.

* Lamas, M. (2014). Ensayo: Entre el estigma y la ley. La interrupción legal del embarazo en df el . Salud Pública, 56, 56-62.

* LeTourneau, K. (2016). El estigma en torno al aborto en el mundo: síntesis de la literatura cualitativa. Un informe técnico para integrantes de la Red Internacional por la Reducción de la Discriminación y el Estigma con relación al Aborto. Inroads.

* Maroto, A. (2009). El trauma post aborto. Un mito creado por sectores conservadores. En: Interrupción terapéutica del embarazo: aportes para la reflexión. Colectiva por el derecho a decidir.

* Morán-Faundes, J. M. (2015). Cuatro focos analíticos en América Latina. En: S. Ramos, editor. Investigación sobre aborto en América Latina y el Caribe. Una agenda renovada para informar políticas públicas e incidencia. Centro de Estudios de Estado y Sociedad-CEDES. 
"Estigma hacia el aborto y sus consecuencias: acciones para reducirlo"

Fabiola Orihuela-Cortés y Ma. Luisa Marván

Vol. 22, Núm. 4, julio-agosto 2021

Revista Digital Universitaria

- O'Donell, A., O'Carrol, T., y Toole, N. (2018). Internalized stigma and stigma-Related isolation predict women's psychological distress and physical health symptoms post-abortion. Psychology of women quarterly, 1-15.

* Rincón, M. y Mazo, S. (2014). El derecho a decidir de las mujeres iobjeción de conciencia, estigma o discriminación? Tejiendo Saberes. Católicas por el Derecho a Decidir, 27, 22-23.

- Shellenberg, K., Moore, A., Bankole, A., Juárez, F., Kehinde, A., Palomino, N., Zeba Sathar. Z., Singh. S. y Tsui, A. (2011). Social stigma and disclosure about induced abortion: results from an exploratory study. Global Public Health: an International Journal for research, policy and practice, 6 (sup 1), 11-1125.

Sigh, S., Remez, L., Sedgh, G., Kwok, L., y Onda, T. (2018). Abortion wordlwide 2017. Uneven Progress and Unequal Access. Instituto Guttmacher.

* Sorhaindo, A., Karver, T., Karver, J., y García, S. (2016). Constructing a validated scale to measure community-level abortion stigma in Mexico. Contraception, 93, 421-431

* Sorhaindo, A., Juárez- Ramírez, C., Díaz Olavarrieta, C., Aldaz, E., Mejía, M. C. y García, S. (2014). Qualitative evidence on abortion stigma from Mexico City and five states in Mexico. Women \& Health, 54 (7), 622-640 Dol: https://doi.org/10.1080 /03630242.2014.919983

* Zamberlin, N. (2015). Estigma y Aborto. En: S. Ramos, editor. Investigación sobre aborto en América Latina y el Caribe. Una agenda renovada para informar políticas públicas e incidencia. Centro de Estudios de Estado y Sociedad-CEDES/Ciudad de México: Population Council.

\section{Sitios de interés}

- Católicas por el Derecho a Decidir: https://www.catolicasmexico.org/i/

* Campaña "Otra mirada Católica del aborto": http://otramirada.catolicasmexico. org/

Católicadas: https://www.youtube.com/watch?v=P8LIW_BhXrl

* Ipas México https://www.ipasmexico.org/

INROADS: https://www.endabortionstigma.org/acerca-de-inroads

* adAS Apoyo Emocional: Facebook adas Apoyo Emocional.

DDSER: https://www.ddeser.org/

* Fondo María: https://www.fondomaria.org/

- Campaña "YO aborto, yo acompaño, yo transformo": https://bit.ly/35Z4EqA

* Campaña "Hablar es chido": http://hablareschido.mx/ 
"Estigma hacia el aborto y sus consecuencias: acciones para reducirlo" Fabiola Orihuela-Cortés y Ma. Luisa Marván Vol. 22, Núm. 4, julio-agosto 2021 Revista Digital Universitaria

- Cortometraje "Era yo, otra vez": https://www.youtube.com/watch?v=GemEK6jIYIk

Focos: https://www.focos.org.mx/focos/

\section{Cómo CITAR ESTE ARTículo}

* Orihuela Cortés, Fabiola y Marván, Ma. Luisa. (2021, julio-agosto). Estigma hacia el aborto y sus consecuencias: acciones para reducirlo. Revista Digital Universitaria (RDU), 22(4). http://doi.org/10.22201/cuaieed.16076079e.2021.22.4.7 